\title{
EPS System of Tractor Automatic Driving: An Improved LMI with Mixed Sensitivity Control Method
}

\author{
Huanxiao Pang $\mathbb{D}^{1},{ }^{1}$ Guangfei Xu $\mathbb{D}^{1,2}$ Zhicai Song, ${ }^{1}$ Dianbao Zhao $\mathbb{D}^{1},{ }^{1}$ Wenjun Wang $\mathbb{D}^{1}{ }^{1}$ \\ and Peisong Diao ${ }^{1}$ \\ ${ }^{1}$ College of Agricultural Engineering and Food Science, Shandong University of Technology, Zibo 255000, China \\ ${ }^{2}$ Liaocheng Academy of Agricultural Sciences, Liaocheng 252000, China
}

Correspondence should be addressed to Wenjun Wang; wjwang2016@163.com and Peisong Diao; dps2003@163.com

Received 10 February 2021; Revised 28 July 2021; Accepted 3 August 2021; Published 14 August 2021

Academic Editor: Jit S. Mandeep

Copyright ( 92021 Huanxiao Pang et al. This is an open access article distributed under the Creative Commons Attribution License, which permits unrestricted use, distribution, and reproduction in any medium, provided the original work is properly cited.

\begin{abstract}
Electric power steering (EPS) is widely used in tractor automatic driving because of its good operation stability. However, there is a lack of research about solving robust problems and response ability simultaneously when the tractor encounters emergency steering in harsh fields. The traditional robust controller has poor tracking performance and antidisturbance ability when encountering emergency steering. This paper proposes to add the corresponding mixed sensitivity operator to the corresponding performance index in the controller. By adjusting the amplitude of the mixed sensitivity operator, the tracking performance and the speed of disturbance attenuation can be both adjusted for the tractor EPS system. Simulation and hardware in the loop experiments verify the antidisturbance ability of the controller and the torque tracking performance. The results show that the control method has strong robustness and robust stability, which can meet the practical requirements. Also, the power steering characteristic of the $H_{\infty}$ controller with hybrid sensitivity design method is better than that of an unoptimized one, and its robustness is better, and under external pavement interference, the following ability is stronger for the ideal hand torque and the steering is more stable.
\end{abstract}

\section{Introduction}

A tractor is the power source in field operation, its working environment is complex, and often it is used in the rugged field work; therefore, power steering system is essential for tractors [1], and scholars have done many kinds of research on it.

In the research process, the vehicle stability [2], the model uncertainty, and the interference problems are mainly concerned and various advanced control methods and research schemes are adopted to improve the overall performance of electric power steering (EPS) under different working conditions [3].

The special working environment of tractors determines that the EPS system of tractors needs to consider the motor control strategy under special working conditions. Bei et al. [4] designed the motor control strategy including the compensation of return-to-normal process, the compensation of emergency avoidance process, the compensation of full-load large-angle turning, and other special steering conditions. However, the influence of random interference and measurement noise on EPS system is not considered. In the research process, the influence of random interference and sensor noise on EPS control cannot be ignored. Therefore, robust control methods have been applied to the research of EPS. Zhao et al. [5] designed a hybrid $H_{2} / H_{\infty}$ robust controller considering tire nonlinearity and external interference. A displacement and force coupling control design for active front steering (AFS) system of vehicle is proposed in their paper. The robustness is controlled by $H_{\infty}$, and the performance is monitored by $\mathrm{H}_{2}$. He et al. [6] put forward a rolling time-domain control method. A robust $H_{\infty}$ coordination control scheme is developed based on linear matrix inequality (LMI) $[7,8]$. It is proposed to simultaneously suppress lateral path-tracking deviation while maintaining autonomous vehicle stability under dynamic driving situations at handling limits. 
However, many control systems including EPS need to deal with multiobjective constraint problem, which is difficult for the aforementioned algorithms. As LMI can take all control targets into consideration by establishing Lyapunov function $[9,10]$, a series of control method based on LMI is proposed. Menhour et al. [11] proposed a two-PID control method based on LMI theory. The controllers are based on LMI theory stabilizing optimal and on linear time-invariant models of the vehicle, obtained around its nominal operating point. The different gains of both PID controllers are managed by a switch depending on the velocity. It can get good control effectiveness, but the author lacks the influence of uncertainty on the controller. Therefore, this paper proposes an LMI control method based on mixed sensitivity operator and applies it to the EPS system. It can be used for tractor power steering under special conditions and solving the influence of random interference and sensor noise on EPS system so that the EPS control strategy of tractors has better operation performance and safety performance.

This paper is arranged as follows. In Section 2, the space model of EPS is established; in Section 3, the controller is designed based on the LMI method; then in Section 4, the simulation analysis is carried out, and the problems of the controller based on the LMI method are found; in Section 5, the existing problems are solved, and the LMI method with mixed sensitivity operator is proposed, and the robust performance is verified; and in Section 6, the algorithm is tested in practice. Section 7 is the conclusion.

\section{Establishment of EPS Model for Tractor}

EPS dynamic modeling is the key of this paper; therefore, we need to carry out mathematical modeling of the main components of the steering power transmission system $[5,12]$. Figure 1 shows the tractor steering system. This paper mainly studies the electric power steering system. The following is the mathematical modeling of the main components.

2.1. Torsion Bar Equation. According to the vehicle dynamics, the torsion bar can be regarded as a scale model in the range of stiffness $[11,13]$ :

$$
\begin{aligned}
\delta & =\frac{x_{r}}{r_{p}}, \\
T_{c} & =K_{s}(\alpha-\delta),
\end{aligned}
$$

where $\delta$ is the steering gear angle, $\mathrm{rad} ; x_{r}$ is the steering gear rack displacement, $m ; r_{p}$ is the radius of steering gear, $m ; K_{s}$ is the torsional rigidity; $T_{c}$ is the torsion bar torque, $N$; and $\alpha$ is the steering wheel angle, rad.

2.2. Electrical Machinery Model. The system uses a brushed DC motor. The differential equation can be obtained by Kirchhoff laws of voltage:

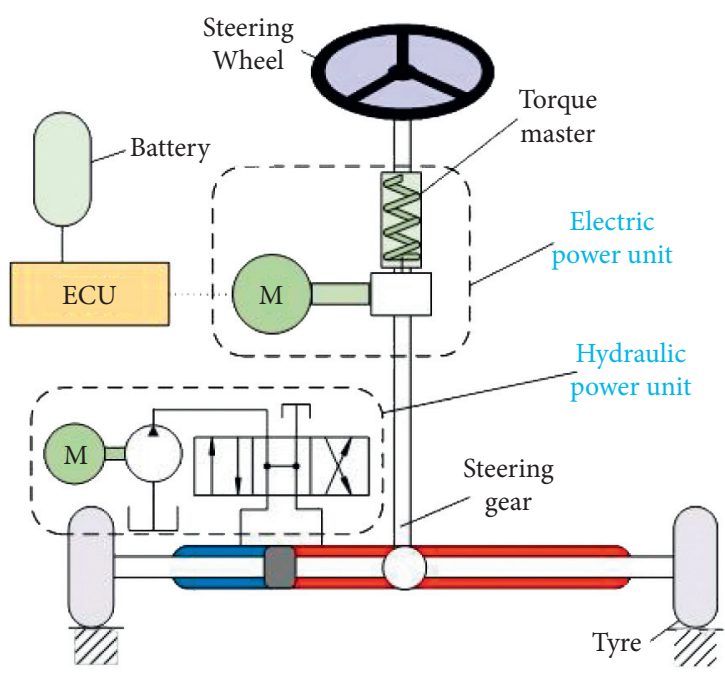

Figure 1: Tractor steering system.

$$
\begin{aligned}
u & =\mathrm{RI}_{a}+L\left(\frac{\mathrm{dI}_{a}}{\mathrm{dt}}\right)+K_{b} \dot{\theta}, \\
\dot{\theta} & =N \dot{\delta}, \\
T_{m} & =K_{a} I_{a},
\end{aligned}
$$

where $u$ is the motor terminal voltage, $\mathrm{V} ; R$ is the armature resistance, $\Omega ; \theta$ is the angle of motor, $\mathrm{rad} N N$ is the drive ratio which is the steering shaft to the motor; $K_{a}$ is the torque coefficient of motor; $K_{b}$ is the coefficient of back electromotive force of motor; $L$ is the inductance coefficient of motor, $H ; I_{a}$ is the motor current, $\mathrm{A}$; and $T_{m}$ is the electromagnetic torque of motor, $\mathrm{N} \mathrm{m}$.

2.3. Tire Model. It is not easy to use the model to accurately build the dynamic characteristics of the tire when the tractor is running, but for the EPS model of the tractor in this paper, it is enough to establish the equation of tire angle and resistance moment. Fujiwara and other experts proposed that the relationship between the steering angle and the tire resistance moment is approximately linear when the vehicle is stationary or at low speed, and the tractor is always at low speed during operation [14], which fully conforms to the aforementioned theory. So the resistance moment of the steering wheel is

$$
T_{\text {tire }}=\theta_{\text {tirel }} K_{\text {tire }},
$$

where $T_{\text {tire }}$ is the steering resisting torque, $\mathrm{Nm}$; $\theta_{\text {tire }}$ is the front wheel actual angle, rad; and $K_{\text {tire }}$ is the proportion coefficient of electric power torque-rotation angle.

It is known from the vehicle system dynamics [15] that there is a difference between the theoretical angle $\theta_{\text {tire }}$ and the actual angle $\theta_{\text {tire } 1}$, which directly affects the return torque of the steering system.

(1) Theoretical angular equation: 


$$
\theta_{\text {tire }}=\frac{\theta_{\text {shaft }}}{N_{2}}
$$

where $\theta_{\text {tire }}$ is the theoretical angle, rad; $\theta_{\text {shaft }}$ is the intermediate shaft angle, rad; and $N_{2}$ is the transmission ratio of steering gear.

(2) Actual angle equation: in the actual angle, when the stiffness coefficient of the intermediate shaft is larger, the deformation angle of the front wheel will be smaller, so

$$
\theta_{\text {tirel }}=\theta_{\text {tire }}-\theta_{\delta}=\theta_{\text {tire }}-\frac{\theta_{\text {tire }}}{K_{\text {rigid }}} \approx \theta_{\text {tire }}
$$

(3) Resistance moment equation of intermediate shaft [16]:

$$
T_{1}=\frac{T_{\text {tire }}}{N_{2}}
$$

where $T_{1}$ is the resistance moment equation of intermediate shaft, $\mathrm{N} \mathrm{m}$.

Therefore, it can be deduced from the aforementioned formula:

$$
T_{1}=\frac{K_{\text {tire }}}{N_{2}^{2}} \theta_{\text {shaft }}
$$

If

$$
\left\{\begin{array}{l}
T_{1}=\frac{K_{\text {tire }}}{N_{2}^{2}} \\
\theta_{\text {shaft }} \approx \delta
\end{array}\right.
$$

then the steering load torque can be expressed as

$$
T_{1}=\delta K_{1} \text {. }
$$

2.4. Controller Model. The controller plays an important role in EPS, which is the basis of the road feel control strategy and assist control strategy. Assist characteristic curve is an important factor of EPS assist characteristic. Figure 2 shows a typical assist characteristic curve. It can be seen from Figure 1 that

$$
i= \begin{cases}0, & 0<T_{c} \leq 1, \\ k(v) T_{c}, & 1<T_{c} \leq 7, \\ 7 k(v), & T_{c}>7,\end{cases}
$$

where $i$ is the motor current and $k(v)$ is the slope at different speeds.

It is also known from the electrical characteristics of DC motor that

$$
i=\frac{\left(u-k_{b} \dot{\theta}\right)}{R} .
$$

We can get

$$
k(v) T_{c}=\frac{\left(u-k_{b} \dot{\theta}\right)}{R} .
$$

Then,

$$
u=k(v) T_{c} R+k_{b} N \dot{\alpha}-k_{b} N \frac{\dot{T}_{c}}{k_{s}} .
$$

Among them,

$$
\begin{aligned}
& k_{d}=-\frac{k_{d} N}{k_{s}}, \\
& k_{p}=k(v) R, \\
& k_{N}=k_{b} N,
\end{aligned}
$$

where $k_{d}$ is the differential coefficient; $k_{p}$ is the proportional coefficient; and $k_{N}$ is the integral coefficient.

The deformation can be

$$
u=k_{p} T_{c}+k_{d} \dot{T}_{c}-k_{N} \dot{\alpha} .
$$

It can be seen that the armature voltage of the motor is determined by the torque and the steering wheel rotating speed, so the electric power steering system mainly regulates the relationship among the steering resistance $T_{1}$, the motor power $T_{m}$, and the driver's hand torque $T_{h}$. Therefore, the controller model can control the motor current or voltage to achieve the coordination of the three.

In conclusion, the nonlinear dynamic model of EPS controlled system is obtained as follows:

(i) Steering column:

$$
I_{h} \ddot{\alpha}+C_{h} \ddot{\alpha}+T_{c}=T_{h}+f_{h}(\alpha, \dot{\alpha}) .
$$

(ii) Output shaft:

$$
I_{e} \ddot{\delta}+C_{e} \dot{\delta}=T_{c}+N T_{\alpha}-T_{l}+f_{e}(\delta, \dot{\delta})
$$

(iii) Assisted motor:

$$
I_{m} \ddot{\theta}+C_{e} \dot{\theta}=T_{m}-T_{\alpha}+f_{m}(\theta, \dot{\theta}) .
$$

(iv) Pinion-and-rack mechanism:

$$
M_{r} \ddot{x}_{r}+C_{r} \dot{x}_{r}+k_{r} x_{r}+T_{R}=\frac{T_{l}}{r_{p}}+f_{r}\left(\dot{x}_{r}, x_{r}\right) .
$$

Among them, $f_{h}(\alpha, \dot{\alpha}), f_{e}(\delta, \dot{\delta}), f_{m}(\theta, \dot{\theta})$, and $f_{r}\left(\dot{x}_{r}, x_{r}\right)$ are time-varying and nonlinear uncertain models.

The deformation can be 


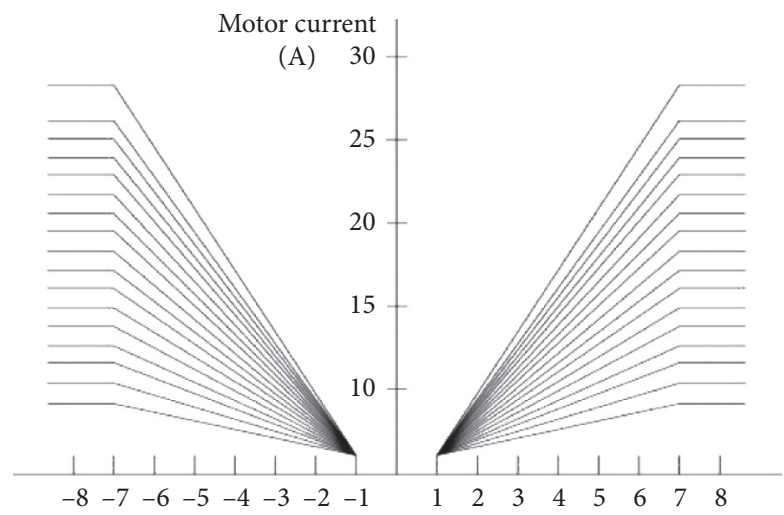

Figure 2: Assist characteristic curve.

$$
\left\{\begin{array}{l}
\ddot{a}=\frac{1}{I_{h}}\left(-K_{s} \alpha-K_{s} \delta+C_{h} \dot{\alpha}+T_{h}+f_{h}(\alpha, \dot{\alpha})\right), \\
\ddot{\theta}=\frac{1}{I_{m}}\left(-K_{m} \theta-\left(C_{m}+\frac{K_{a} K_{b}}{R}\right) \dot{\theta}+K_{m} N \delta+\frac{K_{\alpha}}{R} u+f_{m}(\theta, \dot{\theta})\right) \\
\ddot{x} \\
=\frac{1}{M_{R}}\left(\frac{K_{s}}{r_{p}} \alpha+\frac{N k_{m}}{r_{p}} \theta-\left(K_{r}+\frac{K_{s}}{r_{p}^{2}}+\frac{N^{2} K_{m}}{r_{p}^{2}}\right) x_{r}-C_{R} \dot{x}_{r}+f_{e}\left(\delta, \dot{\delta}, T_{R}\right)-T_{R}\right) \\
\ddot{\delta}=\frac{1}{M_{R} r_{p}}\left(\frac{K_{s}}{r_{p}} \alpha+\frac{N k_{m}}{r_{p}} \theta-\left(K_{r} r_{p}+\frac{K_{s}}{r_{p}}+\frac{N^{2} K_{m}}{r_{p}}\right) x_{r}-C_{R} r_{p} \dot{x}_{r}+f_{e}\left(\delta, \dot{\delta}, T_{R}\right)-T_{R}\right) .
\end{array}\right.
$$

\section{Controller Design Based on LMI Method}

The controller $K(\mathrm{~s})$ is designed through the EPS mathematical model, and the $H_{\infty}$ robust control theory is combined to realize the $H_{\infty}$ robust control standardization of the EPS controlled closed-loop system model [17-19].

The state equation of the system can be obtained as follows:

$$
\left\{\begin{array}{l}
\dot{x}=A x+B_{1} w+B_{2} u, \\
z=C_{1} x+D_{11} w+D_{12} u, \\
y=C_{2} x+D_{21} w+D_{22} u, \\
u=k y .
\end{array}\right.
$$

Take state variables as $x=(\alpha, \delta, \theta, \dot{\alpha}, \dot{\delta}, \dot{\theta})$. The control input is $u=[u] \in R^{n}$. The road signal input is $w=\left[\begin{array}{lll}d_{\delta} & T_{h} & T_{R}\end{array}\right] \in R^{q} . d \delta$ is sensor noise. $T_{R}$ is external disturbance. The control output is $z=\left[\begin{array}{lll}e_{a} & e_{f} & u\end{array}\right]^{T} \in R^{r} . y=\left[T_{c}+d T_{c}\right] \in R^{p}$ is the measured value.
Among them, the coefficient matrices are variable.

$$
A=\left[\begin{array}{cccccc}
0 & 0 & 0 & 1 & 0 & 0 \\
0 & 0 & 0 & 0 & 1 & 0 \\
0 & 0 & 0 & 0 & 0 & 1 \\
-\frac{K_{s}}{I_{h}} & \frac{K_{s}}{I_{h}} & 0 & -\frac{C_{h}}{I_{h}} & 0 & 0 \\
\frac{K_{s}}{M_{R} r_{p}^{2}} & \mathrm{MM} & \frac{\mathrm{NK}_{m}}{M_{R} r_{p}} & 0 & -\frac{C_{R}}{M_{R}} & 0 \\
0 & k_{m} N & -\frac{K_{s}}{I_{m}} & 0 & 0 & \mathrm{HH}
\end{array}\right]
$$




$$
\begin{aligned}
& \mathrm{MM}=-\frac{1}{M_{R} r_{p}}\left(K_{r} r_{p}+\frac{K_{s}}{r_{p}}+\frac{N^{2} K_{m}}{r_{p}}\right), \\
& \mathrm{HH}=-\frac{C_{m} R+K_{a} K_{b}}{I_{m} R}, \\
& B_{1}=\left[\begin{array}{ccc}
0 & 0 & 0 \\
0 & 0 & 0 \\
0 & 0 & 0 \\
0 & \frac{1}{I_{h}} & 0 \\
0 & 0 & -\frac{1}{M_{R} r_{p}} \\
0 & 0 & 0
\end{array}\right],
\end{aligned}
$$

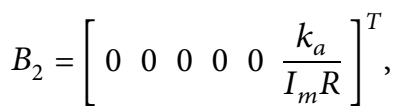

$$
\begin{aligned}
& D_{11}=\left[\begin{array}{ccc}
0 & 0 & 0 \\
0 & 0 & -k_{f} r_{p} \\
0 & 0 & 0
\end{array}\right] \text {, } \\
& D_{12}=\left[\begin{array}{lll}
0 & 0 & 1
\end{array}\right]^{T}, \\
& C_{1}=\left[\begin{array}{ccc}
k_{a} k(s) k_{s} & k_{s} & 0 \\
N k_{m}-k_{a} k(s) k_{s} & -\left(k_{s}+k_{s} k_{r} r_{p}^{2}\right) & 0 \\
-k_{m} & 0 & 0 \\
0 & 0 & 0 \\
0 & -k_{s} c_{r} r_{p}^{2} & 0 \\
-c_{m} & 0 & 0
\end{array}\right]^{T} \\
& C_{2}=\left[\begin{array}{llllll}
k_{s}-k_{s} & 0 & 0 & 0 & 0
\end{array}\right] \text {, } \\
& D_{21}=\left[\begin{array}{lll}
1 & 0 & 0
\end{array}\right] \text {, } \\
& D_{22}=0 \text {. }
\end{aligned}
$$

Formula (23) is the uncertain space state equation based on $H_{\infty}$ robust control, as shown in Figure 3.

$$
\left[\begin{array}{l}
z \\
y
\end{array}\right]=P\left[\begin{array}{l}
w \\
u
\end{array}\right]=\left[\begin{array}{ll}
p_{11} & p_{12} \\
p_{21} & p_{22}
\end{array}\right]\left[\begin{array}{l}
w \\
u
\end{array}\right] .
$$

The output and input of $H_{\infty}$ standardized control system can be transformed into formula (24).

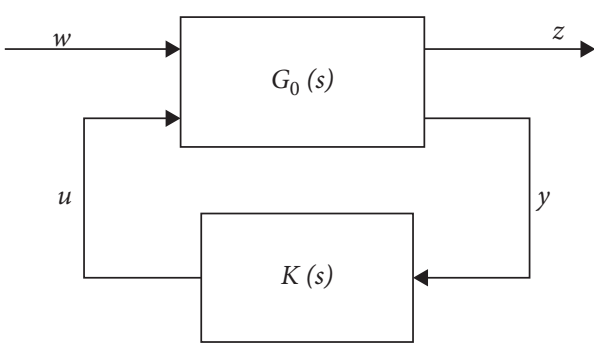

FiguRE 3: $H_{\infty}$ standardized control model.

Using the conversion algorithm of frequency transfer function representation and state space representation, the following can be obtained: the expression of the transfer relationship from the external disturbance $w=\left[d \delta T_{h} T_{R}\right]^{T}$ to the evaluation index $z=\left[e_{a} e_{f}-u\right]^{T}$ of the controlled system is $T_{z w}$. Then, the following control objective based on the standard $H_{\infty}$ control model is to minimize $T_{z w}$ on the basis of solving the $K$ of the controller, that is, the influence of external interference on the evaluation index of the controlled system is reduced to the lowest point.

Let the desired controller $K$ be [20]

$$
\left\{\begin{array}{l}
\dot{x}_{k}=A_{k} x_{k}+B_{k} y \\
u=C_{k} x_{k}+D_{k} y
\end{array}\right.
$$

where the control space of the controller is $x_{k} \in R^{n \times k}$ and $A_{k}$, $B_{k}, C_{k}$, and $D_{k}$ are the parameter space matrices of the controlled system controller to be determined.

From the model of the controlled system (formula (21)) and its controller (formula (25)), the closed-loop system expression of the controlled object can be obtained as follows:

$$
\left\{\begin{array}{l}
\dot{x}_{c}=A_{c 1} x_{c}+B_{c 1} w \\
u=C_{c 1} x_{c}+D_{c 1} w
\end{array}\right.
$$

where $x_{c}=\left[x^{T} x_{k}^{T}\right]^{T}$ is the space state of the controlled object. It is converted to a matrix format of

$$
\left[\begin{array}{ll}
A_{c 1} & B_{c 1} \\
C_{c 1} & D_{c 1}
\end{array}\right]=\left[\begin{array}{cc}
A_{0} & B_{0} \\
C_{0} & D_{11}
\end{array}\right]+\left[\begin{array}{c}
\bar{B} \\
\bar{D}_{12}
\end{array}\right] K\left[\begin{array}{cc}
\bar{C} & \bar{D}_{21}
\end{array}\right]
$$

where $K=\left[\begin{array}{cc}A_{k} & B_{k} \\ C_{k} & D_{k}\end{array}\right]$

Therefore,

$$
\left[\begin{array}{ccc}
A_{0} & B_{0} & \bar{B} \\
C_{0} & D_{11} & \bar{D}_{12} \\
\bar{C} & \bar{D}_{21} & K^{T}
\end{array}\right]=\left[\begin{array}{ccccc}
A & 0 & B_{1} & B_{2} & 0 \\
0 & 0 & 0 & 0 & I \\
C & 0 & D_{11} & D_{12} & 0 \\
C & 0 & D_{21} & D_{k}^{T} & D_{k}^{T} \\
0 & I & 0 & C_{k}^{T} & A_{k}^{T}
\end{array}\right] .
$$

The design requirement of $H_{\infty}$ robust controller is to find $K$, so that the closed-loop expression of the controlled plant (formula (25)) can satisfy the controlled system model (formula (21)) [21]. Assuming that the model of the controlled system and its controller have been determined, the 
sufficient and necessary condition for the controller of the controlled system to be an $H_{\infty}$ controller is that there is a symmetric positive definite matrix $X_{c 1}$ such that the following formula holds:

$$
\left[\begin{array}{ccc}
A_{c 1}^{T} X_{c 1}+A_{c 1} X_{c 1} & X_{c 1} B_{c 1} & C_{c 1}^{T} \\
B_{c 1}^{T} X_{c 1} & -I & D_{c 1}^{T} \\
C_{c 1} & D_{c 1} & -I
\end{array}\right]<0 .
$$

From the controlled system model (formula (21)) and its controller (formula (24)), we can see that formula (28) is the LMI problem. According to the matrix theory, BMI problem can be transformed into LMI problem.

Let

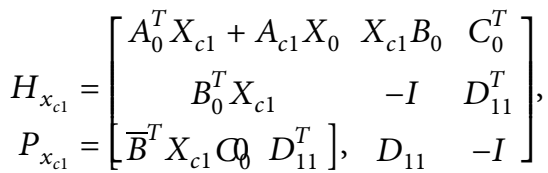

$$
\begin{aligned}
& Q=\left[\begin{array}{lll}
\bar{C} & \bar{D}_{21} & 0
\end{array}\right] \text {. }
\end{aligned}
$$

Then formula (28) can be transformed into

$$
H_{x_{c 1}}+P_{x_{c 1}}^{T} K Q+Q^{T} K^{T} P_{x_{c 1}}<0 .
$$

Set

$$
X_{c 1}, T_{x_{c 1}}=\left[\begin{array}{ccc}
A_{0} X_{c 1}^{-1}+A_{c 1} X_{0} & B_{0} & X_{c 1}^{-1} C_{0}^{T} \\
B_{0}^{T} & -I & D_{11}^{T} \\
C_{0} X_{c 1}^{-1} & D_{11} & -I
\end{array}\right] .
$$

Then the matrix model of the model parameters of the controlled system can be obtained as follows:

$$
P=\left[\begin{array}{lll}
\bar{B}^{T} & 0 & \bar{D}_{12}^{T}
\end{array}\right] .
$$

If $X_{c 1}>0$, then

$$
\begin{gathered}
N_{P_{x c 1}}^{T} H_{x c 1} N_{P_{x c 1}}<0, \\
N_{Q}^{T} T_{x c 1} N_{P}<0 .
\end{gathered}
$$

From the aforementioned study, we can draw the following conclusions: if there is a symmetric positive definite matrix $X_{c 1}$ and

$$
\begin{gathered}
N_{P}^{T} T_{x c 1} N_{P}<0, \\
N_{Q}^{T} H_{x c 1} N_{Q}<0,
\end{gathered}
$$

then there is an $n_{k}$-order $H_{\infty}$ controller for the controlled system model (formula (21)).

If $n_{\mathrm{k}}$ and $n$ are the order of $H_{\infty}$ controller and system model, respectively, $X_{c 1}$ is a real symmetric matrix of $\left(n+n_{k}\right) \times\left(n+n_{k}\right)$ dimension. If $X$ and $Y$ are $n \times n$-dimensional symmetric matrices, the matrix variables $X_{c 1}$ and $X_{c 1}{ }^{-1}$ can be expressed as

$$
\begin{aligned}
X_{c 1} & =\left[\begin{array}{cc}
X & X_{2} \\
X_{2}^{T} & X_{3}
\end{array}\right], \\
X_{c 1}^{-1} & =\left[\begin{array}{cc}
Y & Y_{2} \\
Y_{2}^{T} & Y_{3}
\end{array}\right] .
\end{aligned}
$$

From this, $N_{P}^{T} T_{x c 1} N_{P}<0$ and $N_{Q}^{T} H_{x c 1} N_{Q}<0$ is equivalent to

$$
\begin{aligned}
& {\left[\begin{array}{cc}
N_{c} & 0 \\
0 & I
\end{array}\right]\left[\begin{array}{ccc}
A Y+Y A^{T} & Y C_{1}^{T} & B_{1} \\
C_{1} Y & -I & D_{11} \\
B_{1}^{T} C_{1} & D_{11} & -I
\end{array}\right]\left[\begin{array}{cc}
N_{c} & 0 \\
0 & I
\end{array}\right]<0,} \\
& {\left[\begin{array}{cc}
N_{0} & 0 \\
0 & I
\end{array}\right]\left[\begin{array}{ccc}
A^{T} X+X A & X B & C_{1}^{T} \\
B_{1}^{T} X & -I & D_{11} \\
C_{1} & D_{11} & -I
\end{array}\right]\left[\begin{array}{cc}
N_{0} & 0 \\
0 & I
\end{array}\right]<0,}
\end{aligned}
$$

where $N_{0}$ and $N_{c}$ are matrices with any set of base vectors in kernel space $\operatorname{ker}\left(\left[C_{2} D_{21}\right]\right)$ and $\operatorname{ker}\left(\left[C_{2} D_{21}\right]\right)$ as column vectors, respectively. Feasp, the solver of LMI software package in MATLAB engineering, is used to obtain the feasible solutions of $X$ and $Y$ in the above equation. If $n^{k}$ is a positive integer, $X$ and $Y$ are symmetric positive definite matrices given in $R^{n \times n}$ when

$$
\begin{gathered}
{\left[\begin{array}{cc}
X & I \\
I & Y
\end{array}\right]>0,} \\
\operatorname{rank}\left[\begin{array}{cc}
X & I \\
I & Y
\end{array}\right] \leq n+n_{k} .
\end{gathered}
$$

Formula (39) makes the matrix $X_{2}, Y_{2} \in R^{n \times n k}$ and the positive definite symmetric matrix $X_{3}, Y_{3} \in R^{n \mathrm{kx} n \mathrm{k}}$ satisfy

$$
\begin{aligned}
& {\left[\begin{array}{cc}
X & X_{2} \\
X_{2}^{T} & X_{3}
\end{array}\right]>0} \\
& {\left[\begin{array}{cc}
X & X_{2} \\
X_{2}^{T} & X_{3}
\end{array}\right]^{-1}=\left[\begin{array}{cc}
Y & Y_{2} \\
Y_{2}^{T} & Y_{3}
\end{array}\right] .}
\end{aligned}
$$

Therefore, formulas (37) and (38) have solutions, and their solutions $X$ and $Y$ satisfy equation (39). Then $X_{c 1}$ composed of $X$ and $Y$ satisfies equation (36). If the order of $H_{\infty}$ controller has $n_{k} \geq n$ (as $\operatorname{rank}\left[\begin{array}{cc}X & I \\ I & Y\end{array}\right] \leq 2 n$ ), then its rank can satisfy equation (39). The feasible solution of $X_{c 1}$ is obtained. By substituting this solution into formula (29), the LMI containing only $K$ can be obtained. The Feasp solver in the toolbox is used to obtain the feasible solution $K$ of linear matrix inequality, and finally, the controller is obtained.

\section{Problems in Simulation Based on LMI}

Based on robust control theory, the control objective of EPS uncertain controlled system is designed to evaluate whether it can meet the designed performance to ensure the stability of the controlled system. Table 1 shows the choice of simulation experiment parameters.

The assist torque deviation is the difference between the ideal assist torque $T_{a}^{*}$ and the actual assist torque $T_{a}$ [22]. 
TABLE 1: Engineering parameters of EPS system components.

\begin{tabular}{|c|c|c|c|}
\hline Project & Parameter & Symbol & Values \\
\hline \multirow{6}{*}{ Motor parameter } & Moment of inertia of motor $\left(\mathrm{kg} \mathrm{m}^{2}\right)$ & $I_{m}$ & 0.00018 \\
\hline & Viscous damping coefficient of motor $(\mathrm{N} \mathrm{m} \mathrm{s} / \mathrm{rad})$ & $C_{m}$ & 0.003399 \\
\hline & Resistance $(\Omega)$ & $R$ & $1 / 6$ \\
\hline & Back EMF constant $\left(\mathrm{V} / \mathrm{rad}^{-1}\right)$ & $K_{d}$ & 0.057 \\
\hline & Electromagnetic torque constant $(\mathrm{N} \mathrm{m} / \mathrm{A})$ & $K_{a}$ & 0.04 \\
\hline & Rigidity coefficient of motor $\left(\mathrm{N} \mathrm{M} / \mathrm{rad}^{-1}\right)$ & $K_{m}$ & 125 \\
\hline \multirow{3}{*}{ Steering wheel and column parameters } & Moment of inertia $\left(\mathrm{kg} / \mathrm{m}^{2}\right)$ & $I_{h}$ & 0.0012 \\
\hline & Viscosity damping coefficient ( $\mathrm{N} \mathrm{m} \mathrm{s/rad)}$ & $C_{h}$ & 0.261 \\
\hline & Torsional stiffness of torsion bar $(\mathrm{N} \mathrm{m} / \mathrm{rad})$ & $K_{s}$ & 84.33 \\
\hline \multirow{3}{*}{ Output shaft parameters } & Moment of inertia $\left(\mathrm{kg} / \mathrm{m}^{2}\right)$ & $I_{e}$ & 0.001 \\
\hline & Viscosity damping coefficient ( $\mathrm{N} \mathrm{m} \mathrm{s/rad)}$ & $C_{e}$ & 0.031 \\
\hline & Reduction ratio of worm gear reducer & $N$ & 16.5 \\
\hline \multirow{3}{*}{ Rack and pinion parameters } & Mass (kg) & $M_{r}$ & 0.89 \\
\hline & Viscosity damping coefficient $(\mathrm{Ns})$ & $C_{r}$ & 200 \\
\hline & Steering pinion radius $(\mathrm{m})$ & $r_{p}$ & 0.007 \\
\hline
\end{tabular}

The main reason is that there is a difference between the actual torque and the measured torque due to the hysteresis effect caused by nonlinear mechanical force transmission, component damping, and inertia in the steering system [23]. Torque tracking performance is an important performance of EPS. Therefore, the controller can reduce the torque deviation as much as possible to improve the tracking performance of the whole steering control system. In other words, the designed controller must be able to control the torque deviation $e_{a}=T_{a}-T_{a}{ }^{*}$ as small as possible under the uncertainty of road and human disturbance [24]. The following is a simulation study of $e_{a}$ response to road interference force $T_{R}$ and driver input hand torque $T_{h}$.

Figure 4 shows the response curve of $e_{a}$ to driver input hand torque $T_{h}$ unit step. It can be seen from Figure 5 that when the driver inputs unit $T_{h}$, the EPS assist torque deviation $e_{a}$ will appear a transient process of following. About 0.05 seconds after $T_{h}$ is applied, $e_{\mathrm{a}}$ will reach a maximum following deviation with a peak value of $9.5 \%$ and then decrease sharply. After 2 to 3 seconds of transient time, the steady assist torque deviation is about $1.8 \%$. From the $e_{a}$ to $T_{h}$ unit step response curve, the peak value of assist torque deviation is small, and the stable assist torque deviation is also small. The response effect of EPS assist torque deviation to $T_{h}$ can meet the requirements of EPS controller stability control objectives. From the time effect of stability, the transient process from response to stability takes a long time, and the normal transient time should be less than 2 seconds. Therefore, this obviously cannot meet the sensitivity requirements of EPS in fast steering.

As shown in Figure 5, the driver's input $T_{h}$ is a sine wave at two different frequencies. In Figure 5(a), the frequency of $T_{\mathrm{h}}$ is a low-frequency sine wave of $2 \mathrm{~Hz}$, and the assist torque deviation $e_{\mathrm{a}}$ keeps the synchronous frequency change with the driver's input $T_{h}$, and the phase is the same. In Figure 5(b), the frequency of $T_{h}$ is a $20 \mathrm{~Hz}$ high-frequency sine wave, the power torque deviation $e_{a}$ and the driver input $T_{h}$ cannot keep the synchronous frequency change, and the phase lags behind the phase of $T_{h}$. Therefore, the controller cannot meet the sensitivity requirements of fast steering.

\section{LMI Control Method with Mixed Sensitivity}

From the aforementioned simulation analysis, it can be seen that the $e_{\mathrm{a}}$ following sensitivity cannot meet the designed control requirements when the controller inputs high frequency. In order to further improve the control quality of the designed controller, LMI algorithm with mixed sensitivity operator is adopted. The integrated control structure is shown in Figure 6.

\subsection{Simulation and Evaluation of EPS Assist Torque Deviation} Control. Since the system sensitivity $S_{0}(\mathrm{~s})$ is essentially the closed-loop system transfer function matrix between the output $y$ of the controlled system and the disturbance signal $d$, the designed controller requires that the singular value of $S_{0}(\mathrm{~s})$ is very small, so the ability of the controller to suppress the disturbance will be robust. Similarly, for the EPS closedloop system, $S_{0}(\mathrm{~s})$ is also the transfer function matrix between the torque error $e$ and the ideal input $r$. Therefore, $S_{0}(\mathrm{~s})$ will determine the tracking performance of the assist torque error $e$. The smaller the singular value of $S_{0}(\mathrm{~s})$, the stronger the robustness of the system controller and the better the tracking performance. To adjust a certain performance index of the controlled system, it is very important to determine the corresponding weighting function. The mixed sensitivity operator $W(\mathrm{~s})$ is introduced to adjust the sensitivity of the assist following deviation $e_{a}$. The amplitude of $W(\mathrm{~s})$ can be used to adjust the tracking performance of $e_{a}$ and the attenuation speed of disturbance. Therefore, the control quality of $e_{a}$ can be improved by adjusting the weighting function $W_{s 1}(\mathrm{~s})$ of $e_{\mathrm{a}}$.

As shown in Figure 7, the unit step response curve of $e_{\mathrm{a}}$ to $T_{h}$ when the weighting function is $W_{s 1}(s)=40 / s+0.5$. It can be seen from the figure that the EPS assist torque deviation will have a following transient process. About 0.05 seconds after $T_{h}$ is applied, $e_{a}$ will reach a maximum follow-up deviation of $3.8 \%$ and then decrease sharply. After 0.1 -second transient time, the deviation of stable assist torque is about $0.026 \%$. Compared with Figure 5, the control quality of $e_{a}$ by 


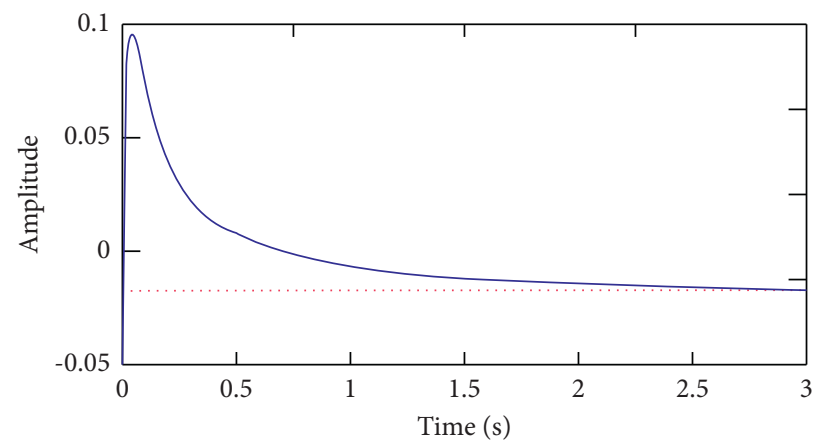

FIgURE 4: Unit step response curve of $e_{a}$ to $T_{h}$.

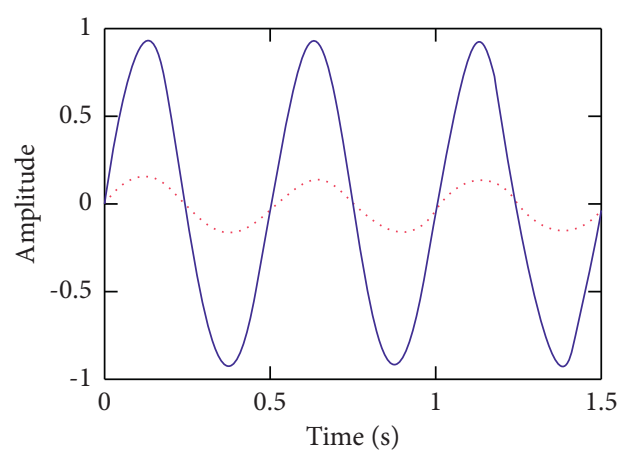

(a)

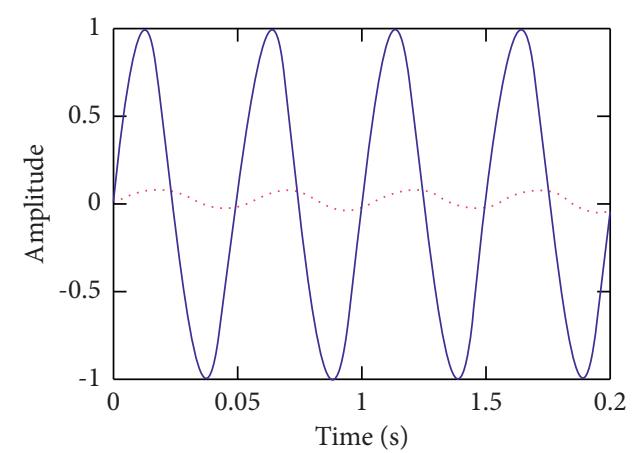

(b)

Figure 5: Sine response curve of $e_{a}$ to $T_{h}$. (a) $2 \mathrm{~Hz}$ response of input hand torque and (b) $20 \mathrm{~Hz}$ response of input hand torque.

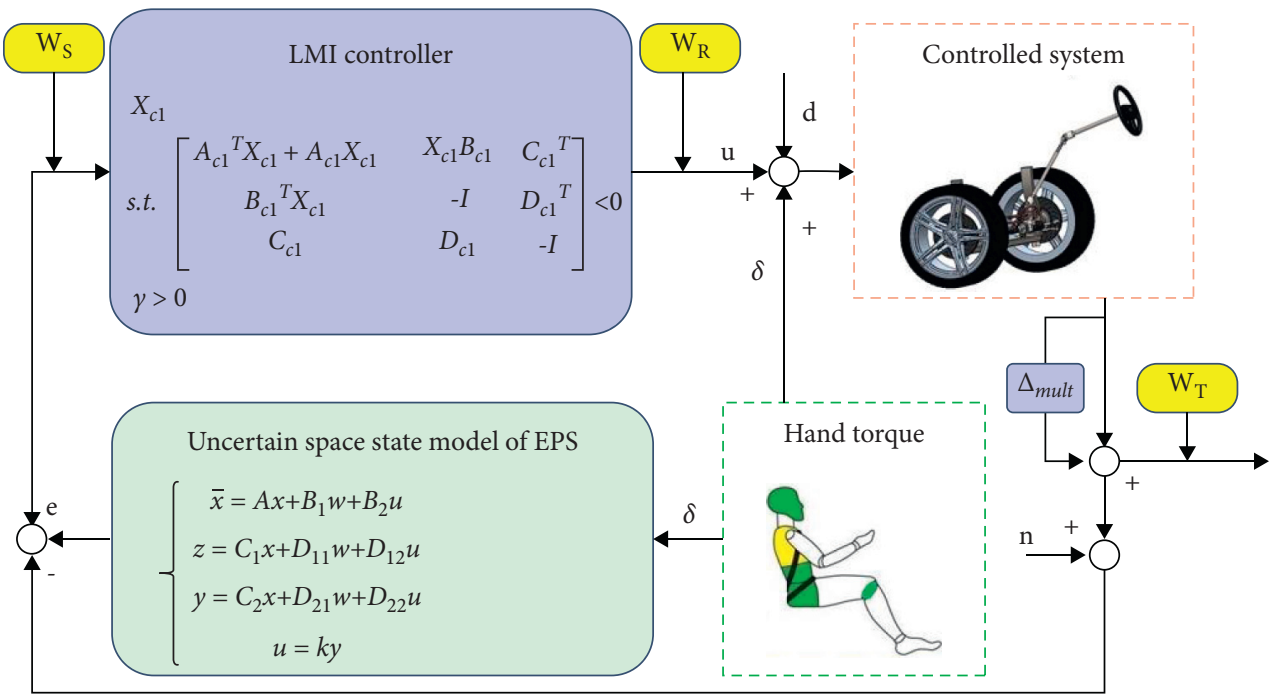

Figure 6: The control structure.

the controller has been significantly improved in both response speed and phase synchronization. Therefore, the control performance of the controller model based on $H_{\infty}$ robust control theory has been significantly improved.

\subsection{Simulation and Evaluation of EPS Road Feel Control.} EPS road feel control steering system control performance index is another important performance index, which is related to the driver's comfort in the process of driving. In the actual driving state, steering resisting torque $T_{1}$ and road interference $T_{R}$ will change with the change of road conditions. It is significant to study this performance index for safe and stable driving.

The control effect of EPS road feel is evaluated by road feel error $e_{f}$. Figure 8 shows the unit step response curve of $e_{f}$ to $T_{h}$. In Figure $8(\mathrm{a})$, the mixed sensitivity operator is $W_{s 1}(s)=1 /(s+0.5)$. The peak response of $e_{f}$ to $T_{h}$ unit step 


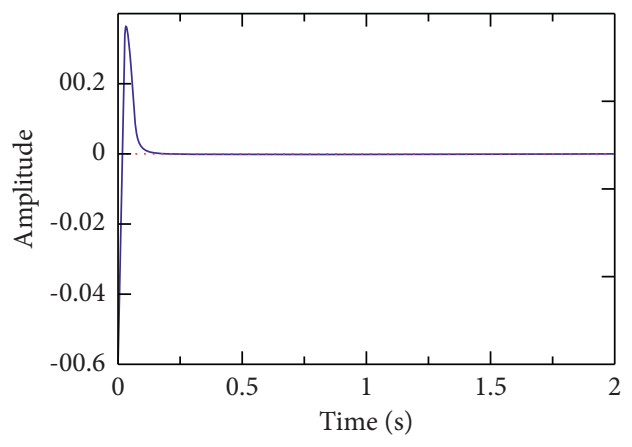

(a)

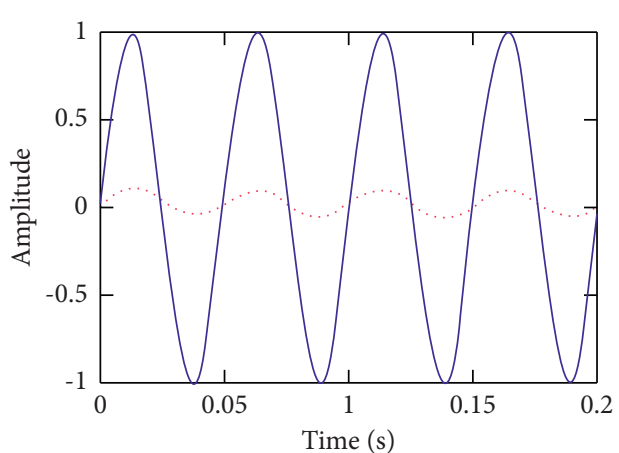

(b)

FIgURE 7: Response curve of $e_{a}$ to $T_{h}$. (a) Unit step and (b) sine wave $(20 \mathrm{~Hz})$.

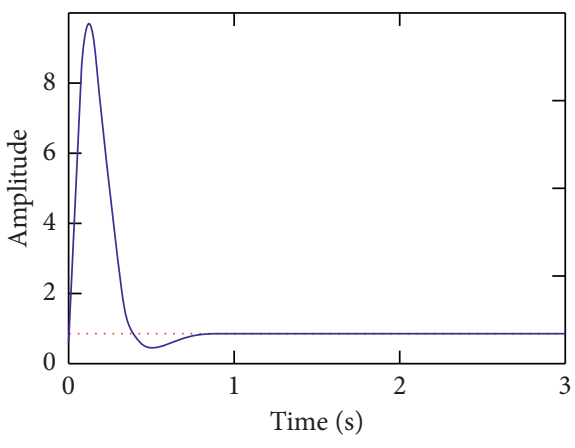

(a)

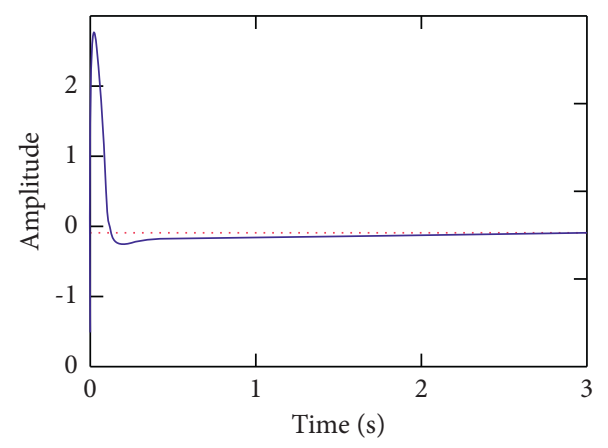

(b)

FIGURE 8: Response curve of $e_{f}$ to $T_{h}$. (a) $W_{s 1}(s)=1 /(s+0.5)$ and (b) $W_{s 1}(s)=100 /(s+0.5)$.

is about 8.7. There is a transient process of 0.8 seconds after $T_{h}$ is applied, and then it goes into steady state, which can meet the stability requirements of the steering system. However, if the response peak value of $e_{f}$ to $T_{h}$ unit step is too large, it will make the driver feel "overshoot" for a very short time, which may cause the oscillation of the steering system. Therefore, it is necessary to adjust the weighting function of $e_{f}$. Adjust the original $W_{s 1}(s)=1 /(s+0.5)$ to $W_{s 1}(s)=100 /(s+0.5)$, and the simulation effect is shown in Figure 8(b). It can be seen from the figure that the peak value of $e_{f}$ response to $T_{h}$ unit step is about 2.2, there is a transient process of 0.6 seconds after $T_{h}$ is applied, and then it enters the steady state. At this time, it can not only meet the stability of EPS steering system but also prevent "overshoot" feeling. By adjusting the parameters of $W_{s 1}(\mathrm{~s})$ weighting function, the control performance of EPS can be improved continuously, so as to realize the smoothness of EPS working state.

\subsection{Control Simulation and Evaluation of EPS Robustness.}

By adjusting the corresponding performance weighting function $W_{S}(\mathrm{~s})$, the performance of the controlled system can be improved to a certain extent without modifying the parameters of the mechanical parts of EPS.

EPS inevitably faces the interference of structural uncertainty. The main structural disturbance factors include steering column vibration caused by uneven road surface, torque disturbance of steering system caused by electromagnetic control system, disturbance of modeling parameters (such as change of system working environment, change of gap between components, wear between components, aging of components, and zero drift of electronic components). The simulation study is a comprehensive evaluation of the stability of the controller model under structural uncertainty disturbance based on $H_{\infty}$ robust control theory. In order to better compare the adjustment of single index with the comprehensive adjustment of robustness, the simulation condition is that except for the selected parameters to be disturbed, the weighted function matrix $W$ and other parameters of the controlled system remain unchanged. By formula,

$$
a=\frac{k_{s}}{I_{h}}
$$

That is, the variation range of torsional stiffness $K_{\mathrm{s}}=84.33 \mathrm{~N} \mathrm{~m} / \mathrm{rad}$ in actual operation is $-27.4 \%$ to $27.4 \%$; the moment of inertia $I_{h}=0.0012 \mathrm{~kg} / \mathrm{m}^{2}$ of steering column and steering wheel in actual operation is $-31.8 \%$ to $31.8 \%$. Figure 9 shows that the unit step response of $e_{a}$ to $T_{h}$ is a curve cluster when the disturbance interval of $a$ is 90275 to 50275. It can be seen from the performance of curve cluster in Figure 9 that $e_{a}$ reaches the peak value in about 0.1 seconds when $T_{h}$ is applied, and the fluctuation range is $0.06 \%$ to $0.094 \%$, and the jitter range relative to its central curve is $-0.02 \%$ to $-0.01 \%$. After the peak value, the curve in the 


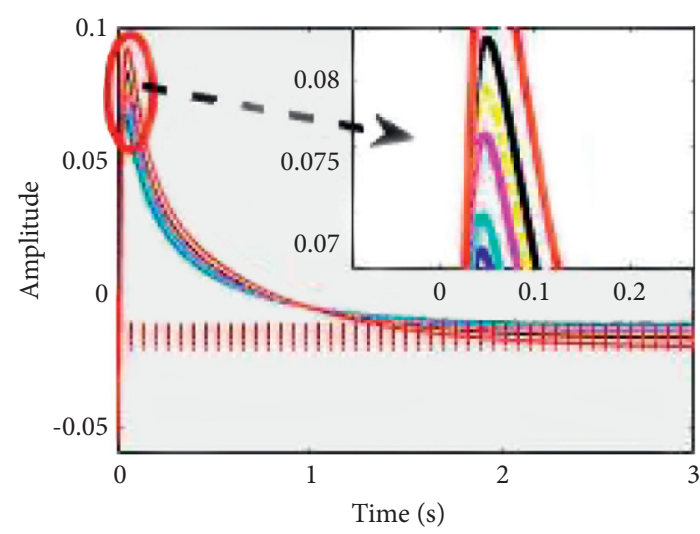

Figure 9: Unit step response curve cluster I of $e_{a}$ to $T_{h}$.

curve cluster drops sharply and tends to be stable in about 1.6 seconds. Therefore, as a closed-loop system, EPS is robust. The research on different parameter weights shows that the robust performance of the whole system is better than before after the $H_{\infty}$ robust controller is applied to the control system.

In the process of simulation, it is very important to adjust the parameters of weighting function. For example, the variation range of parameter $a$ in Figures 9 and 10 is 90275 to 50275 , which reflects the tolerance effect of $T_{h}$ and external interference on $e_{a}$. But the weighting function selected in Figure 9 is $W_{T 1}(s)=(0.01 s+3) /(s+6000)$, while the weighting function selected in Figure 10 is $W_{T 1}(s)=50(s+30) /(s+6000)$. It can be seen from the two figures that the stability accuracy of the controlled system in Figure 10 is worse than that in Figure 9, and the $e_{\mathrm{a}}$ response curve in Figure 10 is relatively divergent than that in Figure 9. In general, the amplitude and follow-up of the response curves in Figures 9 and 10 reflect the robust stability of the controlled system.

EPS controller may produce a certain range of disturbance in the process of its use. The causes may be as follows: first, from the hardware point of view, it may be due to the time shift of physical devices in the process of loading the program; second, from the perspective of software, the transfer function in our design of $H_{\infty}$ controller may be high-order, but in practical application, as hardware operation speed and accuracy cannot keep up with the ideal controller, in this case, we can only reduce the order of the controller algorithm, and then, this also leads to a certain degree of error in the actual controller. So the disturbance of the controller also has the problem of stability. Using the same method, if other conditions are the same, only the uncertainty of the controller itself is changed to $|\Delta|$, so that $|\Delta| \leq 10 \%$, then the generalized controlled system model is as follows:

$$
G_{p}(s)=(1+\Delta) G_{0}(s)
$$

At this time, $e_{a}$ response curve cluster to $T_{h}$ is shown in Figure 11.

It can be seen that the response curve cluster has good stability and robust stability in terms of the convergence of

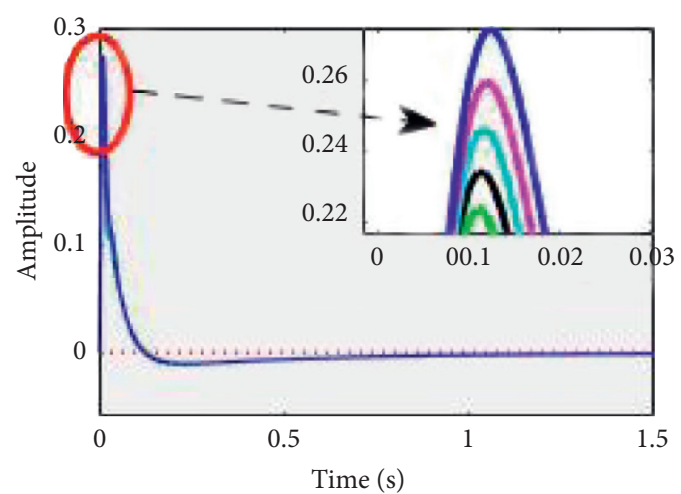

Figure 10: Unit step response curve cluster II of $e_{a}$ to $T_{h}$.

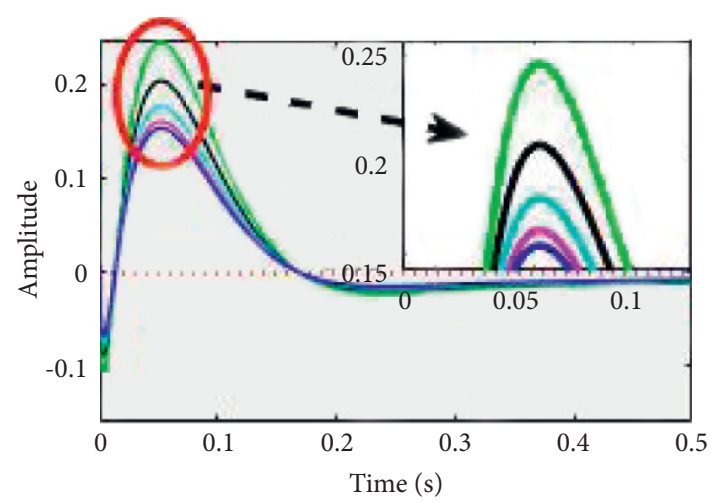

FIgURE 11: Step response from $T_{h}$ to $e_{a}$ with controller uncertainty.

amplitude and final curve and the divergence of curve cluster.

\section{Hardware in the Loop Test}

In the part of simulation test, the robustness of EPS is simulated and evaluated by MATLAB. In this section, the designed controller is embedded into the built-in EPS experimental platform, and the tracking effect between the actual value and the target value is mainly observed, so as to verify the performance of the controller. The EPS hardware in the loop test bed [25] is shown in Figure 12.

Under the condition of in situ simulation, the hand torque is applied on the steering wheel, and the dSPACE software console records the actual current to the target current data under different conditions in real time. The tracking effect between the actual torque and the target torque and between the actual current and the target current is analyzed [26], and then the performance of the controller is verified. The hardware in the loop test results are shown in Figures 13 and 14.

Figures 13 and 14 are the response curves under two different input paths, respectively. From the angle comparison in Figure 13(a), it can be seen that the real angle and the target angle follow well, and the real angle fluctuates about $0.8 \%$. After $50 \mathrm{~ms}$, the real angle reaches a stable state and can always be stable near the expected value. To sum up, when the input changes, although the rotation angle will 

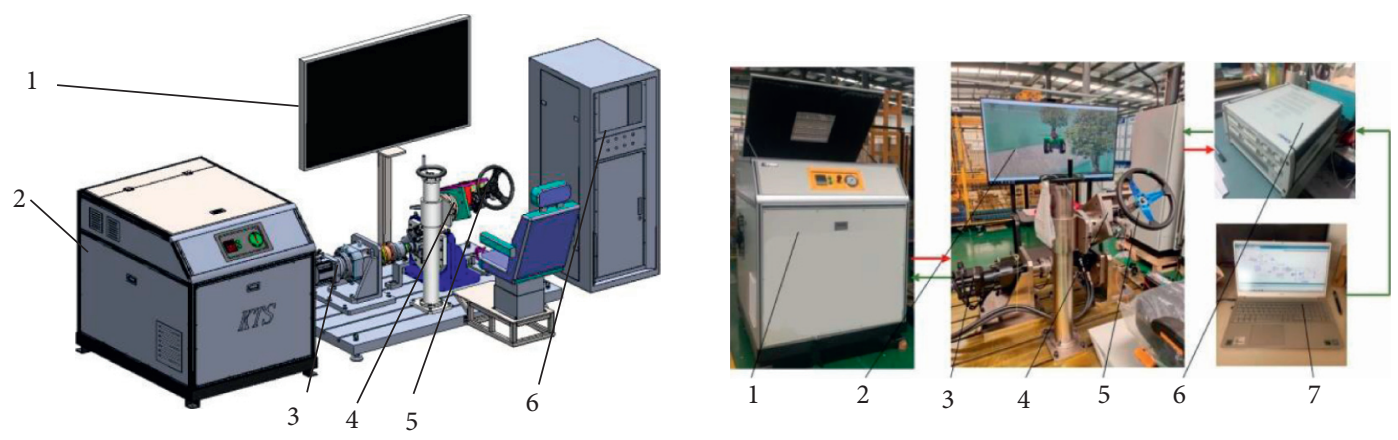

FIGURE 12: EPS hardware in the loop test bed.

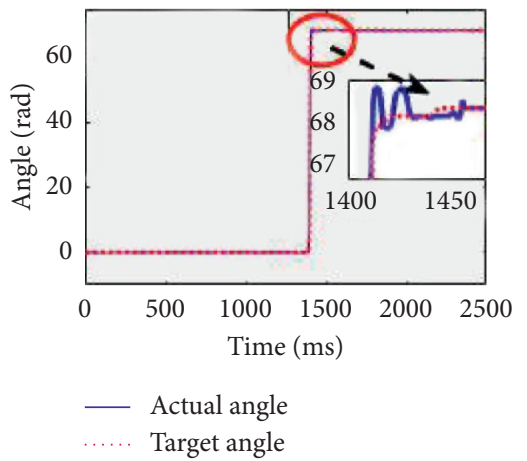

(a)

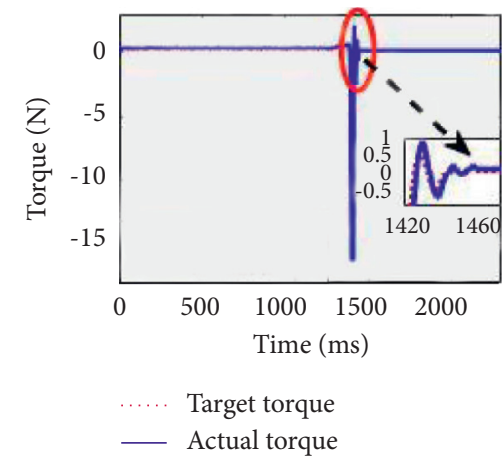

(b)

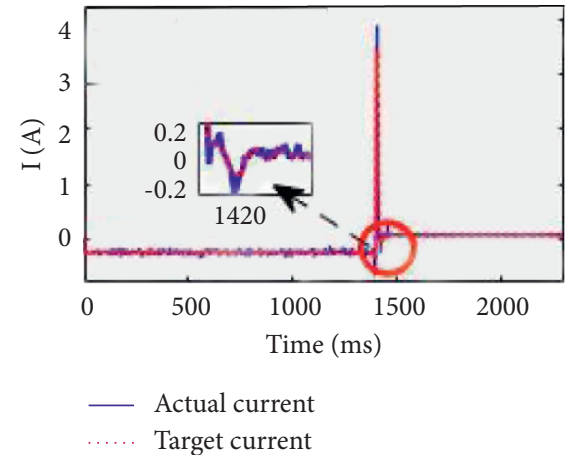

(c)

Figure 13: Response curve under step signal. (a) Angle contrast curve. (b) Torque contrast curve. (c) Current contrast curve.

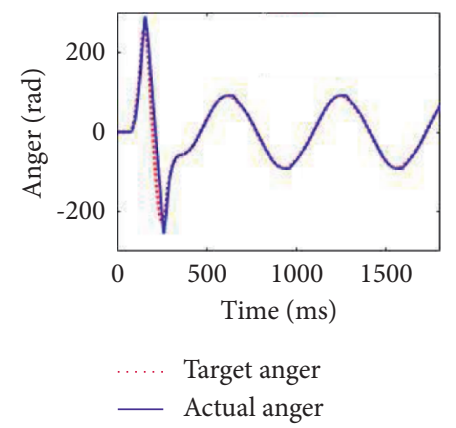

(a)

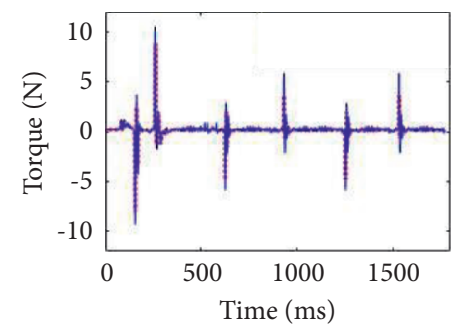

Target torque
- Actual torque

(b)

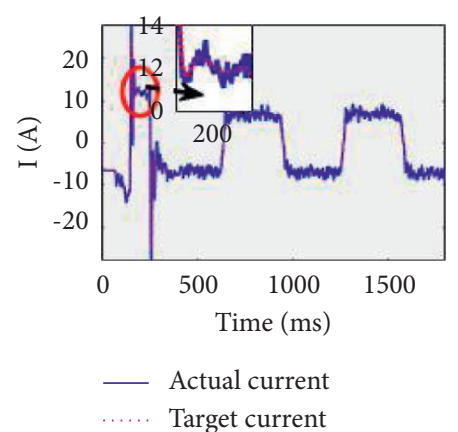

(c)

FIgURE 14: Response curve under sinusoidal signal. (a) Angle contrast curve. (b) Torque contrast curve. (c) Current contrast curve.

fluctuate slightly, the real rotation curve will follow the target curve smooth and stable.

Comparing Figure 13(b) with Figure 13(c) and Figure 14(b) with Figure 14(c), we can see that the torque changes with the change of current, both of them reach the peak value in 1400 and $200 \mathrm{~ms}$, respectively, then there is a small amplitude oscillation, and finally reach the stable value immediately. The algorithm ensures the fast response of the current and the small fluctuation in the target range. The results show that the optimization of the power steering system is good, which can make the driver get less delay road feel and smooth feel, and can make the power motor reach the predetermined torque in a short time.
From the overall test results, the hysteresis between the power steering torque and the target torque is very small, which indicates that the sensitivity of the system is very good. In the comparison diagram of actual angle and target angle, the curve following performance is better, and there is no large fluctuation and overshoot phenomenon, which indicates that the power steering system will not appear steering jitter phenomenon in the actual field work.

\section{Conclusion}

In this article, the EPS controller based on LMI control method with mixed sensitivity operator is designed. First, 
nonlinear dynamic model of EPS controlled system is derived. Then, LMI controller with mixed sensitivity operator is derived, and the controller stability is verified. Simulation is carried out to validate the control effectiveness. The main work of this article mainly has the following aspects:

(a) When the EPS controller is designed with LMI algorithm simply, if the hand torque input frequency becomes larger, the steering wheel angle and steering gear angle cannot keep up with the change of hand torque in amplitude and frequency. It can be concluded that the single LMI controller cannot meet the sensitivity requirements of EPS fast steering.

(b) The LMI controller with mixed sensitivity operator is designed. The antidisturbance ability of the controller and the control effect of the power steering system are verified by simulation and hardware in the loop experiment. Compared with the simple LMI controller and the LMI controller with mixed sensitivity, the tracking performance of the gear angle to the steering wheel angle is robust. That is to say, in the process of vehicle driving, the tracking performance target of the controlled system can be guaranteed under the changes of road interference, frequency, and amplitude of steering wheel input hand torque.

\section{Data Availability}

All data generated or analyzed during this study are included within the article.

\section{Conflicts of Interest}

The authors declare that they have no conflicts of interest.

\section{Acknowledgments}

The authors greatly appreciate the support from Shandong Province Agricultural Application Technology Innovation Project (SD2019NJ005), Shandong Province Agricultural Machinery Equipment Research and Development Innovation Plan Project (2018YF007), National Key Research and Development Programs (2018YFD0300606-04), Shandong Province Modern Agricultural Industrial System Corn Innovation Team Mechanical Processing Post Expert Project, and Shandong Province Agricultural Major Application Technology Innovation Project (SD2019NJ005).

\section{References}

[1] M. Xu, J. Zhou, and C. Sun, "Control characteristics of EPS system of electric tractor," IAEJ, vol. 28, no. 4, p. 22, 2019.

[2] L. Chen, C. Yuan, and H. Jiang, "A study on the handling stability of vehicles with EPS based on $\mu$ synthesis robust control," Automotive Engineering, vol. 30, no. 8, pp. 705-708+712, 2008.

[3] A.-T. Nguyen, C. Sentouh, and J.-C. Popieul, "Sensor reduction for driver-automation shared steering control via an adaptive authority allocation strategy," IEEE/ASME Transactions on Mechatronics, vol. 23, no. 1, pp. 5-16, 2018.
[4] S. Bei, J. Zhao, H. Liu, L. Sun, and L. Chen, "Automotive EPS motor control strategy including special steering situations," Journal of System Simulation, vol. 21, no. 18, pp. 5886-5891+5895, 2009.

[5] W. Zhao, H. Zhang, and Y. Li, "Displacement and force coupling control design for automotive active front steering system," Mechanical Systems and Signal Processing, vol. 106, pp. 76-93, 2018.

[6] X. He, Y. Liu, K. Yang, J. Wu, and X. Ji, "Robust coordination control of AFS and ARS for autonomous vehicle path tracking and stability," in Proceedings of the IEEE International Conference on Mechatronics and Automation (ICMA), August 2018.

[7] M. Chilali, P. Gahinet, and C. Scherer, "Multi-objective output-feedback control via LMI optimization," IFAC Proceedings Volumes, vol. 29, no. 1, pp. 1691-1696, 1996.

[8] H. D. Tuan and P. Apkarian, "Relaxations of parameterized LMIs with control applications," International Journal of Robust and Nonlinear Control, vol. 9, no. 2, 1999.

[9] A.-T. Nguyen, C. Sentouh, H. Zhang, and J.-C. Popieul, "Fuzzy static output feedback control for path following of autonomous vehicles with transient performance improvements," IEEE Transactions on Intelligent Transportation Systems, vol. 21, no. 7, pp. 3069-3079, 2020.

[10] P. Li, A.-Tu Nguyen, H. Du, Y. Wang, and H. Zhang, "Polytopic LPV approaches for intelligent automotive systems: state of the art and future challenges," Mechanical Systems and Signal Processing, vol. 161, 2021.

[11] L. Menhour, D. Lechner, and A. Charara, "Vehicle steering control based on robust control for high lateral accelerations: experimental evaluation," in Proceedings of the 13th International IEEE Conference on Intelligent Transportation Systems, pp. 19-22, Funchal, Portugal, September 2010.

[12] G. Xu, H. Pang, M. Chen, Z. Song, D. Zhao, and P. Diao, "Design of hardware in loop tractor electro-hydraulic coupling steering test platform," Transactions of the Chinese Society for Agricultural Machinery, vol. S1, pp. 525-534+549, 2020.

[13] W. Kim, Y. S. Son, and C. C. Chung, "Torque-overlay-based robust steering wheel angle control of electrical power steering for a lane-keeping system of automated vehicles," IEEE Transactions on Vehicular Technology, vol. 65, no. 6, pp. 4379-4392, 2016.

[14] Y. Fujiwara and S. Adachi, "Steering assistance system for driver characteristics using gain scheduling control," in Proceedings of the European Control Conference 2015, IEEE, Cambridge, UK, September 2003.

[15] Y. Fan and Y. Lin, Automobile System Dynamics 2017, (Chapter 13), 2th ed. edition, 2017.

[16] Z. Jiang and B. Xiao, "Electric power steering system control strategy based on robust $\mathrm{H}$ control for electric forklift," Mathematical Problems in Engineering, vol. 2018, 2018.

[17] G. Xu, P. Diao, X. He, J. Wu, G. Wang, and C. Wang, "Research on vehicle active steering control based on linear matrix inequality and hardware in the loop test scheme design and implement for active steering," Advances in Mechanical Engineering, vol. 11, no. 11, 2019.

[18] J. Tanagorn and B. David, "Iterative LMI approach to robust static output feedback control of uncertain polynomial systems with bounded actuators," Automatica, vol. 123, 2021.

[19] G. Chesi, "LMI techniques for optimization over polynomials in control: a survey," IEEE Transactions on Automatic Control, vol. 55, no. 11, pp. 2500-2510, 2010. 
[20] T. Jennawasin and D. Banjerdpongchai, "Design of statefeedback control for polynomial systems with quadratic performance criterion and control input constraints," Systems and Control Letters, vol. 1175, pp. 3-59, 2018.

[21] J. Wu, Y. Zhao, X. Ji, Y. Liu, and C. Yin, "A modified structure internal model robust control method for the integration of active front steering and direct yaw moment control," Science China Technological Sciences, vol. 58, no. 1, pp. 75-85, 2015.

[22] H. Du and N. Zang, F. Naghdy, Robust control of vehicle electrorheological suspension subject to measurement noises," Vehicle System Dynamics, vol. 49, pp. 1-2, 2011.

[23] X. Li, X.-P. Zhao, and J. Chen, "Controller design for electric power steering system using T-S fuzzy model approach," International Journal of Automation and Computing, vol. 6, no. 2, pp. 198-203, 2009.

[24] A. B. Elghonemy and A. A. El-Badawy, "Robust H-infinity controller for a single-axis spacecraft rotation," in Proceedings of the 2020 12th International Conference on Electrical Engineering (ICEENG), 2020.

[25] H. Zheng and M. Zhao, "Development a HIL test bench for electrically controlled steering system," SAE 2016 World Congress and Exhibition, vol. 01, no. 51, pp. 1-7, 2016.

[26] H. Wu, G. Xu, J. Wu et al., "EPS current tracking method research based on hybrid sensitivity Ho control algorithm," Journal of Electrical and Computer Engineering, vol. 2018, pp. 1-10, 2018. 